\title{
Chromatic Dispersion Compensation Effect Performance Enhancements Using FBG and EDFA-Wavelength Division Multiplexing Optical Transmission System
}

\author{
Mounia Chakkour, ${ }^{1}$ Otman Aghzout, ${ }^{2}$ Badiaa Ait Ahmed, \\ Fahd Chaoui, ${ }^{1}$ and Mounir El Yakhloufi ${ }^{3}$ \\ ${ }^{1}$ Department of Physics, Faculty of Sciences, UAE, Tetouan, Morocco \\ ${ }^{2}$ Department of Telecommunication, ENSA, LabSIT, FS, Tetouan, Morocco \\ ${ }^{3}$ Condensed Matter Physics Group, Faculty of Sciences, UAE, Tetouan, Morocco \\ Correspondence should be addressed to Mounia Chakkour; chakkourm@gmail.com
}

Received 21 March 2017; Accepted 16 November 2017; Published 14 December 2017

Academic Editor: Sulaiman W. Harun

Copyright ( 2017 Mounia Chakkour et al. This is an open access article distributed under the Creative Commons Attribution License, which permits unrestricted use, distribution, and reproduction in any medium, provided the original work is properly cited.

\begin{abstract}
An optical transmission system using Fiber Bragg Grating (FBG) and Erbium Doped Fiber Amplifier (EDFA) with new proposed model has been analyzed to overcome chromatic dispersion and attenuation phenomena. To evaluate the transmission system performance of the received signals, a simple model of one channel transmission has been developed in the first step. Also, optical fiber length and attenuation coefficient parameters have been investigated in detail to deal with the optimized corresponding parameter values. Results show that the performance of the optimized design parameters is very efficient in terms of output power $(\mathrm{dBm})$, noise figure $(\mathrm{dB})$, gain $(\mathrm{dB})$, and $Q$-Factor. The model of one channel developed previously has been adapted to a complex model of four optical channels multiplexing with different wavelengths. FBG and EDFA have been also added to WDM technology system to enhance the chromatic dispersion and the signal attenuation. Results show that the new model is more efficient in terms of $Q$-Factor and eye diagrams.
\end{abstract}

\section{Introduction}

In recent years, optical fiber communication has become one of the main pillars of modern telecommunications networks that is due to its rapid development and its broad range of applications. Nowadays, these modern telecommunication networks are becoming more and more complex [1, 2]. Optical network that applies WDM is currently widely used in existing telecommunication infrastructures and is expected to play a significant role in next generation networks and the future Internet $[3,4]$. These systems often include multiple signal channels, different topology structure, nonlinear devices, and non-Gaussian noise source, which make their design and analysis quite complex and require highintensity work. When different wavelengths of light pulses propagate in an optical fiber, it undergoes a phenomenon of dispersion which results in a temporal spreading. This leads to errors in receiver and prevent correct interpretation of the received signals. Attenuation occurs as well during the signal propagation, which decrease the signal power during its transmission. To face these problems, many solutions have recently been suggested. The use of FBG is one of the most efficient technologies used in research papers to overcome chromatic dispersion problem due to its low cost and its negligible effect $[5,6]$. Besides, there are several types of amplifiers such as SOA, EDFA, and Raman amplifier that have been introduced to encounter the attenuation effects. However, EDFA still provide better results [7-9]. In this paper, we propose a new model to treat chromatic dispersion and signal attenuation. In the first section, we present a detailed study of one channel transmission system with Fiber Bragg Grating and Erbium Doped Fiber Amplifier. FBG and EDFA have been implemented to optimize the quality of the received signal and to overcome chromatic dispersion 


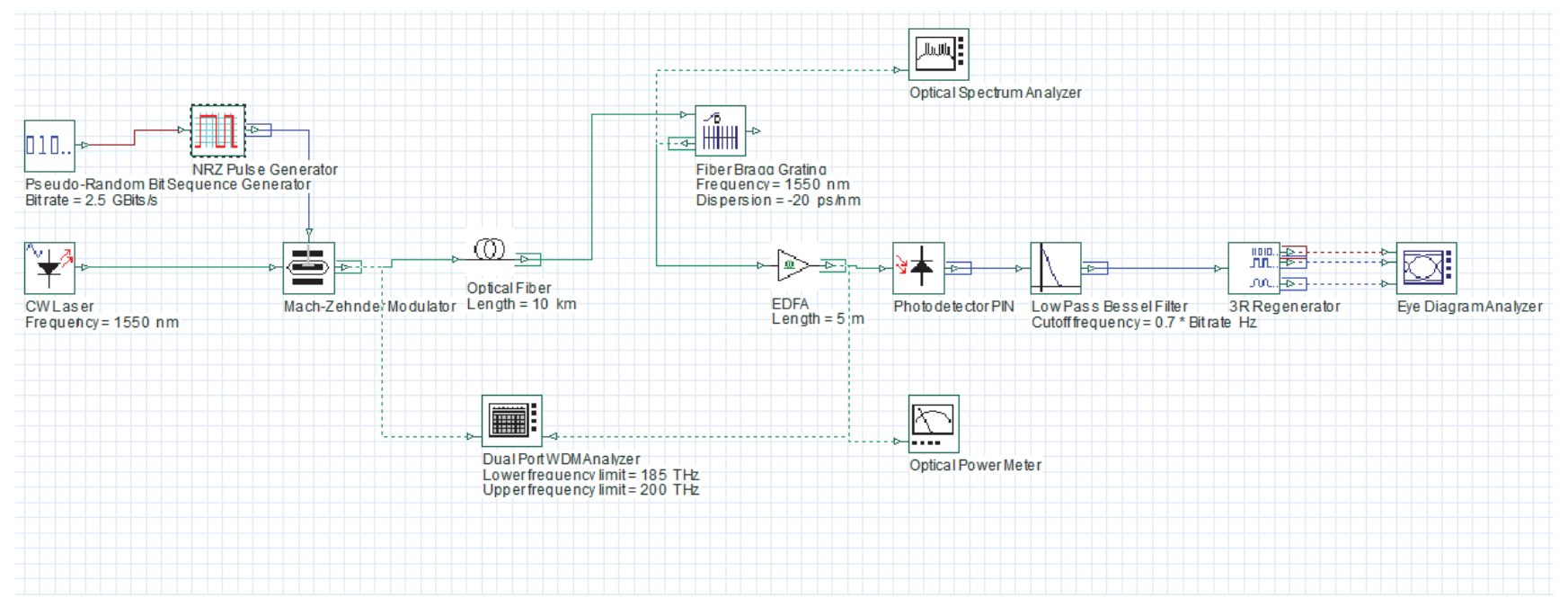

Figure 1: The designed model with one input.

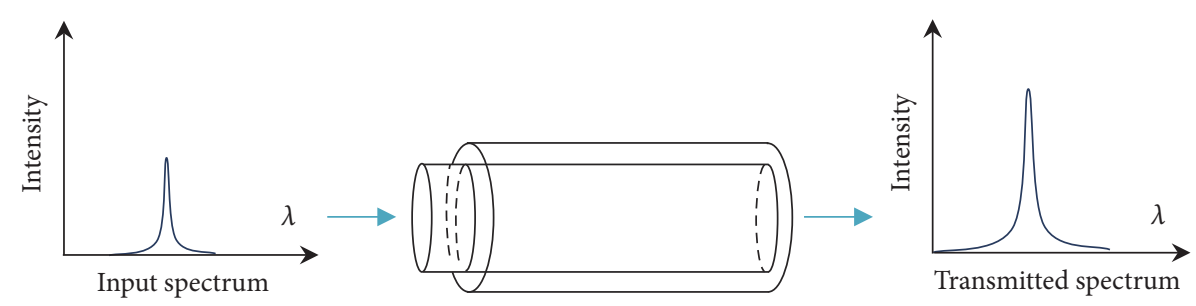

FIgURe 2: Erbium Doped Fiber Amplifier.

and attenuation effects. Then, we compare the transmission system under study using the optimized setting parameters with a previous model proposed by other authors having chirped FBG as dispersion compensator [4-7] in terms of output power $(\mathrm{dBm})$, noise figure $(\mathrm{dB})$, gain $(\mathrm{dB}), \mathrm{Q}$-Factor, and eye diagrams at the received signal. In third section, we apply our model in four optical channels multiplexing with different wavelengths using an EDFA-WDM optical transmission system [9-11]. Finally, we apply and compare with a previous work using the WDM technology to prove more and more the efficiency of our model [12]. The simulation results demonstrate the high efficiency of the developed transmission system.

\section{One Optical Channel Communication System}

As first step, we consider a simple case of one channel optical transmission system as shown in Figure 1. The system consists of three sections: the transmitter, the receiver, and the optical fiber as transmission medium. In the transmission part, we use NRZ pulse generator to provide a controlling bandwidth and pseudo-random bit sequence generator to scramble data signal in terms of bit rates. The input signal is modulated with continuous wave semiconductor laser of $1550 \mathrm{~nm}$ frequency and $5 \mathrm{dBm}$ input power through Mach-Zehnder modulator with $30 \mathrm{~dB}$ of extinction ratio.
To overcome the attenuation problem, we use the Erbium Doped Fiber Amplifier (EDFA) as shown in Figure 2. EDFA is the most often used optical amplifier due to the low loss optical window of silica based fiber. It uses a doped optical fiber as a gain medium to amplify the optical signal [5]. The signal to be amplified and a pump laser are multiplexed into a doped fiber, then it is amplified through interaction with the doping ions.

To face chromatic dispersion, we use Fiber Bragg Grating (FBG) as shown in Figure 3. FBG consists of a periodic modulation of the refractive index in the core of a singlemode optical fiber. The Bragg grating condition satisfies both energy and momentum conservation. The first-order Bragg condition is simplified as follows:

$$
\lambda_{B}=2 n_{\mathrm{eff}} \Lambda
$$

where the Bragg grating wavelength $\lambda_{B}$ is the free space wavelength of the input light reflected from the grating, $n_{\mathrm{eff}}$ is the effective refractive index of the fiber core at the free space center wavelength, and $\Lambda$ is the grating spacing of the FBG [6].

In Figure 4, we show the chromatic dispersion compensation effects on the eye diagrams of the received signal in one channel transmission system. It can be observed that the designed model offers reduced signal distortion and improved eye opening. 


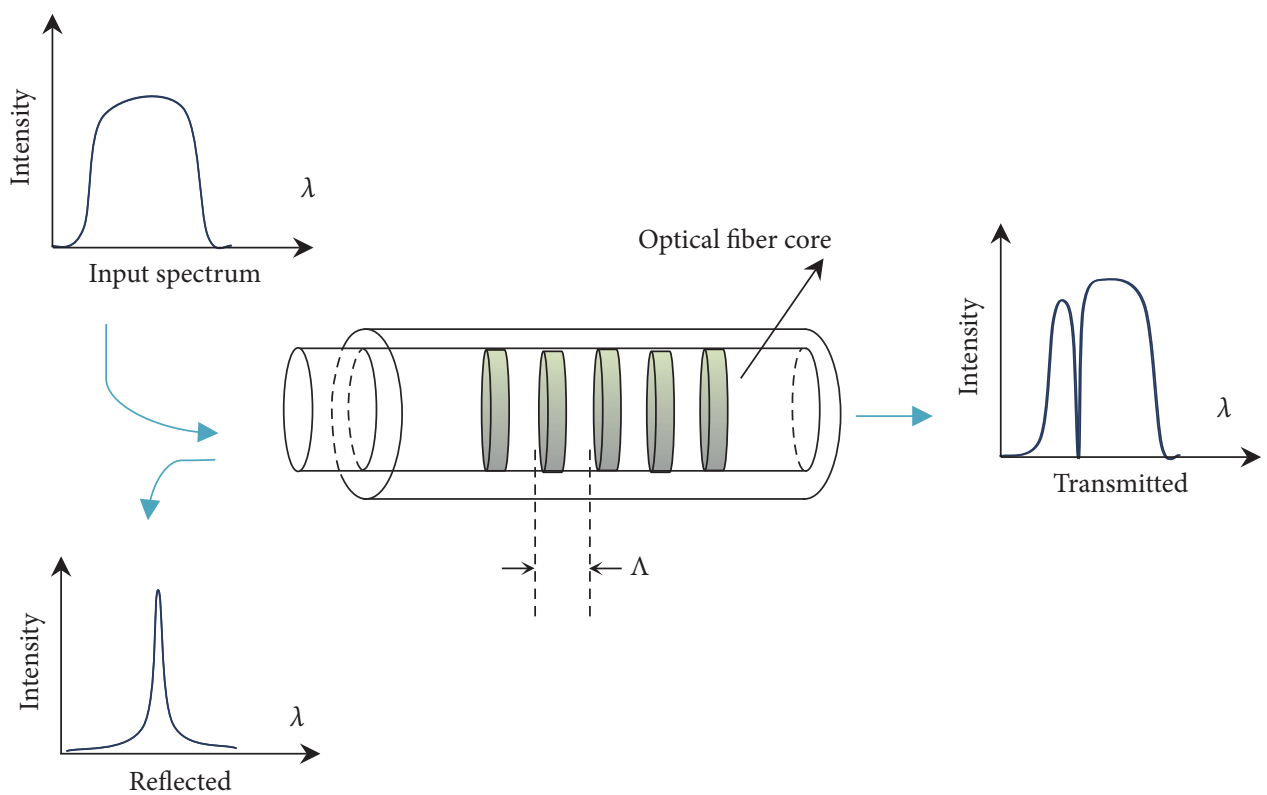

Figure 3: Fiber Bragg Gratings.

TABLE 1: Output readings by varying the OFC length $(\mathrm{Km})$.

\begin{tabular}{lcccc}
\hline OFC length $(\mathrm{Km})$ & Gain $(\mathrm{dB})$ & Noise figure $(\mathrm{dB})$ & Output power $(\mathrm{dBm})$ & Q-Factor \\
\hline 5 & 25.5710 & 4.271 & 16.927 & 243.791 \\
10 & 25.4509 & 5.2754 & 16.807 & 81.465 \\
15 & 25.3029 & 6.3431 & 16.659 & 43.814 \\
20 & 25.1272 & 7.2474 & 16.483 & 33.751 \\
25 & 24.9126 & 8.2467 & 16.274 & 28.023 \\
30 & 24.6708 & 9.2546 & 16.079 & 23.647 \\
\hline
\end{tabular}

2.1. Output Readings of Proposed Model with Varying OFC Length. In this section, we study the impact of the Optical Fiber (OFC) length on the performance of the proposed optical transmission system. Figure 5 shows the eye diagrams for different values of OFC length. By comparing the 6 eye diagrams, we can obviously observe that when OFC length increases the eye closure remains almost the same, which proves the efficiency of the optical network developed in this paper. To evaluate the transmission system performance of the received signals, the $Q$-Factor has been also taken into account in this study. The $Q$-Factor can be easily expressed as follows:

$$
Q=\frac{f_{r}}{\Delta f}
$$

where $f_{r}$ and $\Delta f$ are the resonant frequency and the resonance width or full width at half maximum (FWHM), respectively.

Table 1 represents the results of the output readings at different values of the OFC length of our interest. It can be clearly noticed that the gain, the output power, and $Q$-Factor still relatively the same as the OFC length increases, while the noise figure increases gradually as the fiber length increases.
2.2. Output Readings of Proposed Model with Varying Attenuation Coefficient. We analyze the effect of attenuation coefficient on the performance of the proposed optical transmission system. It is customary to express the loss in units of $\mathrm{dB} / \mathrm{km}$ and thus a loss of $\alpha(\mathrm{dB} / \mathrm{km})$. The loss incurred by propagating down a fiber can be modeled easily as follows:

$$
\alpha=-10 \log _{10}\left(\frac{P_{\text {out }}}{P_{\text {in }}}\right),
$$

where $P_{\text {out }}$ and $P_{\text {in }}$ are the output power of signal and the input power in fiber, respectively [2].

Attenuation coefficient impact on the performance of the proposed transmission system is also studied. By comparing the four eye diagrams obtained in Figure 6, it can be clearly seen that the eye opening is higher at $0.2(\mathrm{~dB} / \mathrm{Km})$ and $1(\mathrm{~dB} / \mathrm{Km})$.

Gain, noise figure, output power, and Q-Factor at different values of attenuation coefficient are illustrated in Figure 7. It can be observed that the smallest value of noise figure and the best value of gain, output power, and $\mathrm{Q}$-Factor are at $0.2(\mathrm{~dB} / \mathrm{Km})$. Then, we consider that $0.2(\mathrm{~dB} / \mathrm{Km})$ is the most proper attenuation coefficient to be used in the proposed mode. 

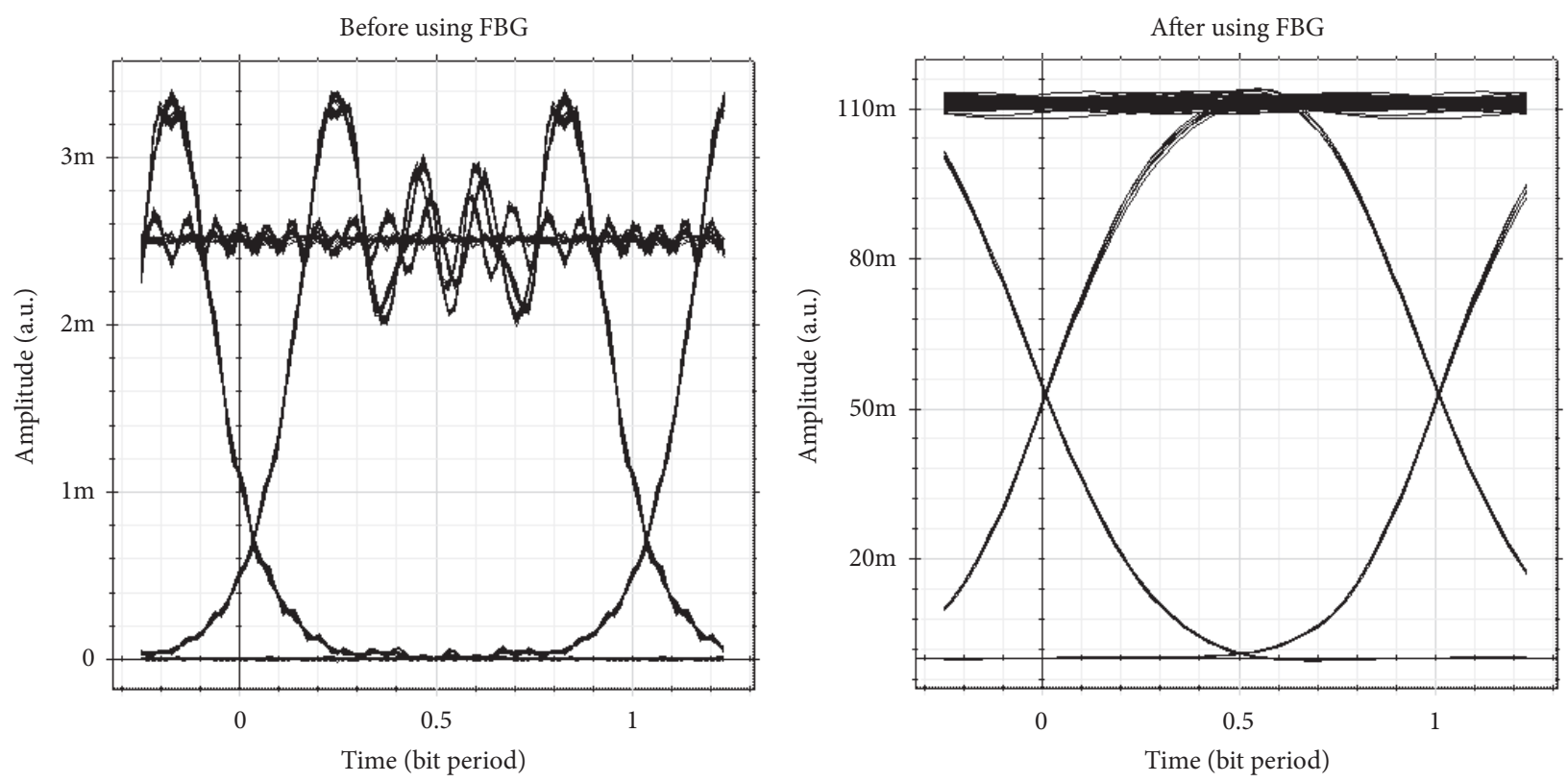

FIGURE 4: Eye diagram before using FBG and after using FBG.
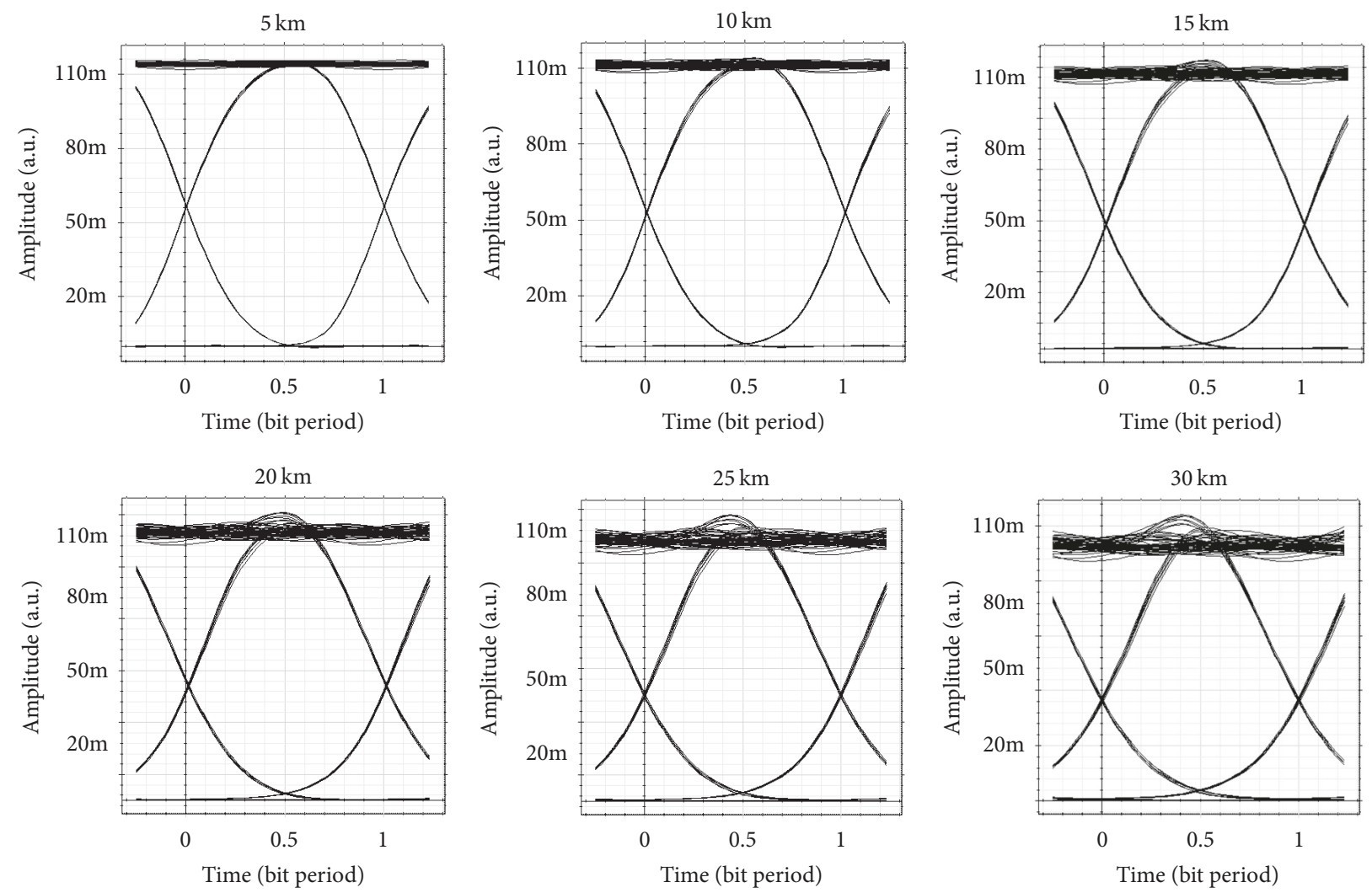

FIGURE 5: Eye diagrams for different values of OFC length. 

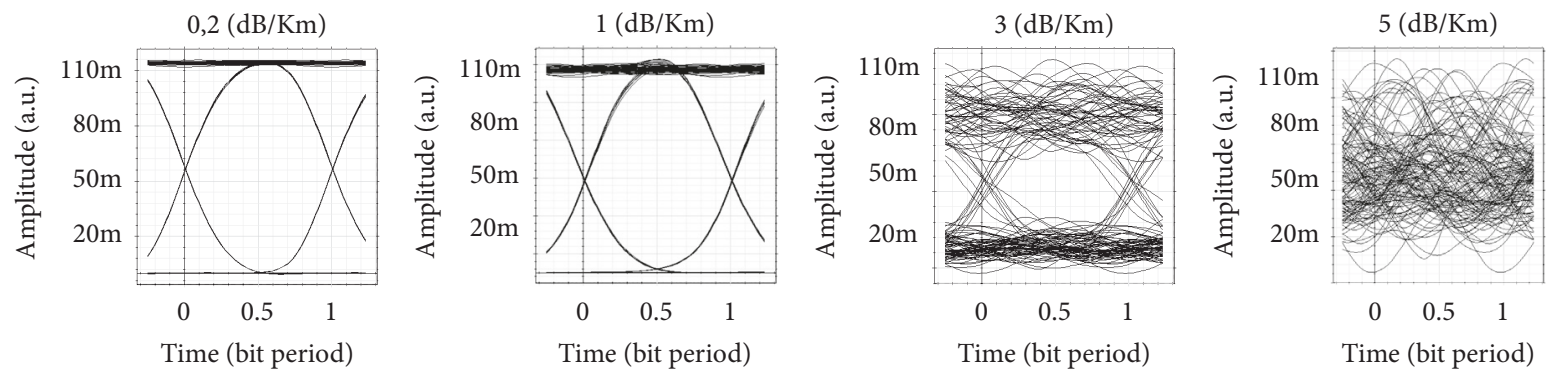

Figure 6: Eye diagrams for different values of attenuation coefficient $(\mathrm{dB} / \mathrm{Km})$.

TABLE 2: Comparison parameters with proposed model and previous models.

\begin{tabular}{lcccc}
\hline Parameters & Without & With (EDFA + uniform FBG) & [5] (EDFA + chirped FBG) & [6] (2EDFA + chirp FBG) \\
\hline Gain $(\mathrm{dB})$ & -0.999 & 25.4509 & 12.239332 & 14.429858 \\
Noise figure $(\mathrm{dB})$ & 0.999 & 5.27549 & 12.2316 & 8.08123 \\
Output power $(\mathrm{dBm})$ & 1.710 & 16.807 & 6.168 & 12.205 \\
Q-Factor & 30.024 & 81.465 & - & 50.2712 \\
\hline
\end{tabular}

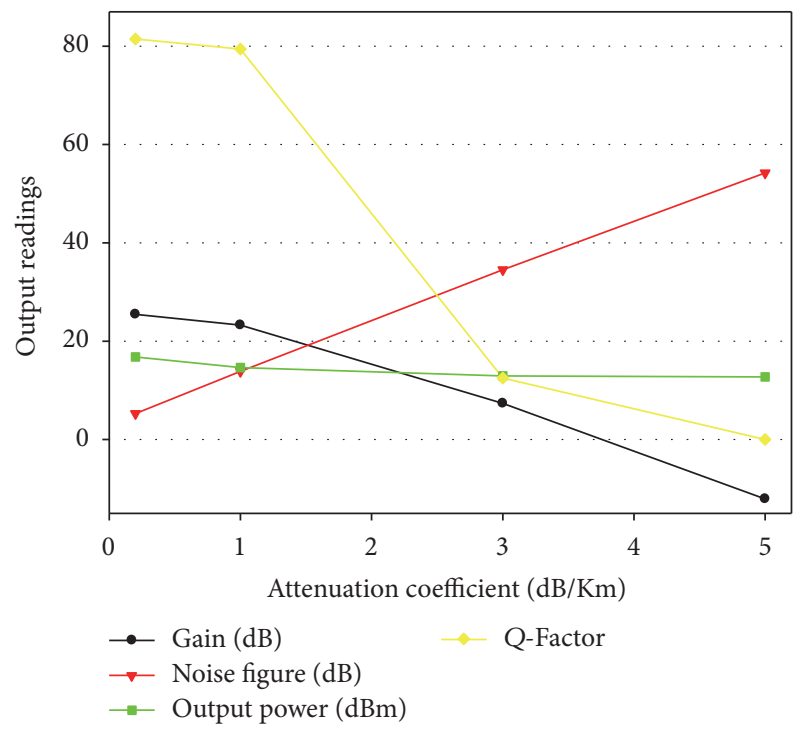

FIGURE 7: Output readings by varying the attenuation coefficient.

Table 2 shows the comparison between the proposed model and the model without EDFA and FBG, respectively. On the other hand, our model using EDFA and FBG has been also compared with $[5,6]$, as shown in Table 2; the proposed model presents many advantages in terms of gain, output power, and signal quality performance with lower noise figure.

\section{WDM Transmission System Model with Four Optical Channels}

Wavelength Division Multiplexing (WDM) is an important progress in the development history of optical fiber communication technology. The basic principle of the WDM is that light signals with different wavelengths are put together at first and then coupled to fiber optic cable lines in the same fibers for transmission $[1,2]$. At last the receiver separates the different wavelengths by signal processing, restores the original signal, and sends them to different terminals as shown in Figure 8.

To validate the efficiency of the developed method in optical transmission network, we have applied the Wavelength Division Multiplexing technology in the model under study. Figure 9 shows the optical WDM transmission system with four input signal channels.

Table 3 shows the simulation results of the proposed model of four optical channels multiplexing. It can be clearly observed that despite adapting the developed model to a complex model using Wavelength Division Multiplexing technology, simulation results remain valid, which means that our model offers reduced signal distortion and improved the gain and the output power.

\section{Performance Comparison}

To prove more and more the reliability of the developed model, we compare the performance of our developed method with a previous work proposed by other authors. So we have integrated FBG and EDFA in WDM optical transmission system [12] as shown in Figure 10. The effect of channel spacing is analyzed by propagating two channels on the same pumped Raman fiber. A silica fiber Raman amplifier pumped at a wavelength of $1451.2 \mathrm{~nm}$ with pump power $1000(\mathrm{~mW})$ is designed. The Raman fiber length is kept at $10 \mathrm{~km}$. The bit rate is kept at $10 \mathrm{~Gb} / \mathrm{s}$ for $0(\mathrm{dBm})$ input signal source. The quality factor is investigated for different values channel spacing [12].

The eye diagrams are represented in Figure 11. As it can be clearly shown, the eye opening is higher when the proposed model is implemented.

In Figure 12, we compare Q-Factor in our model with optical transmission system using WDM technology for two 


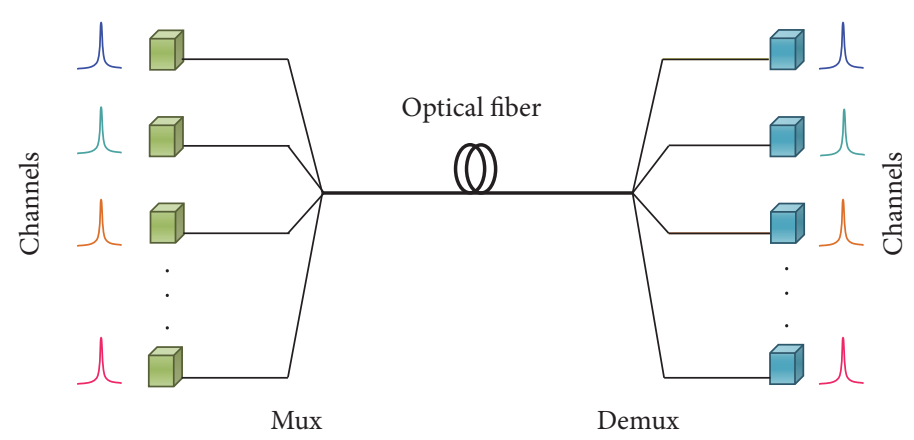

Figure 8: Principle of Wavelength Division Multiplexing (WDM).

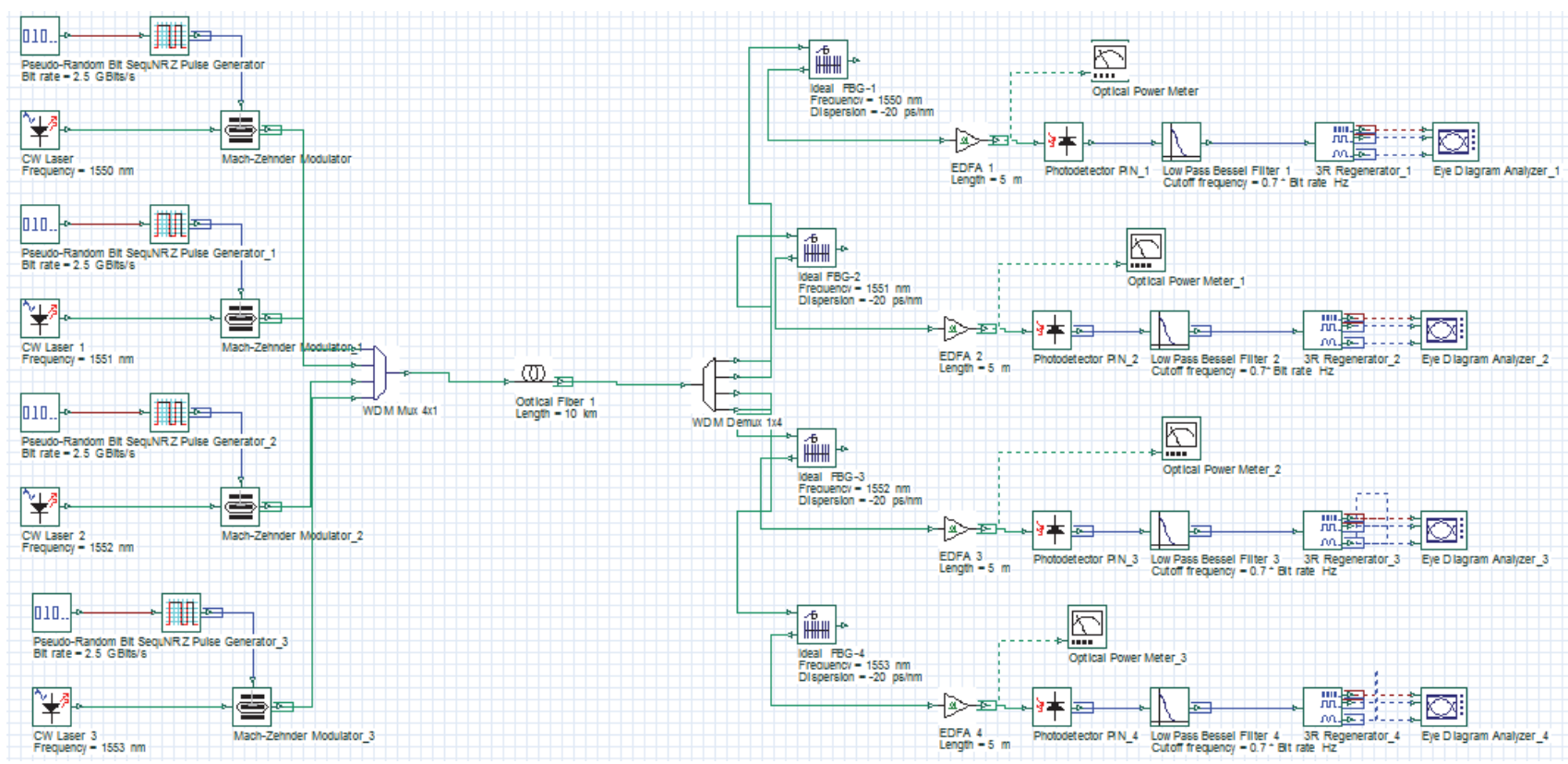

FIGURE 9: Proposed model of four channels WDM transmission system.

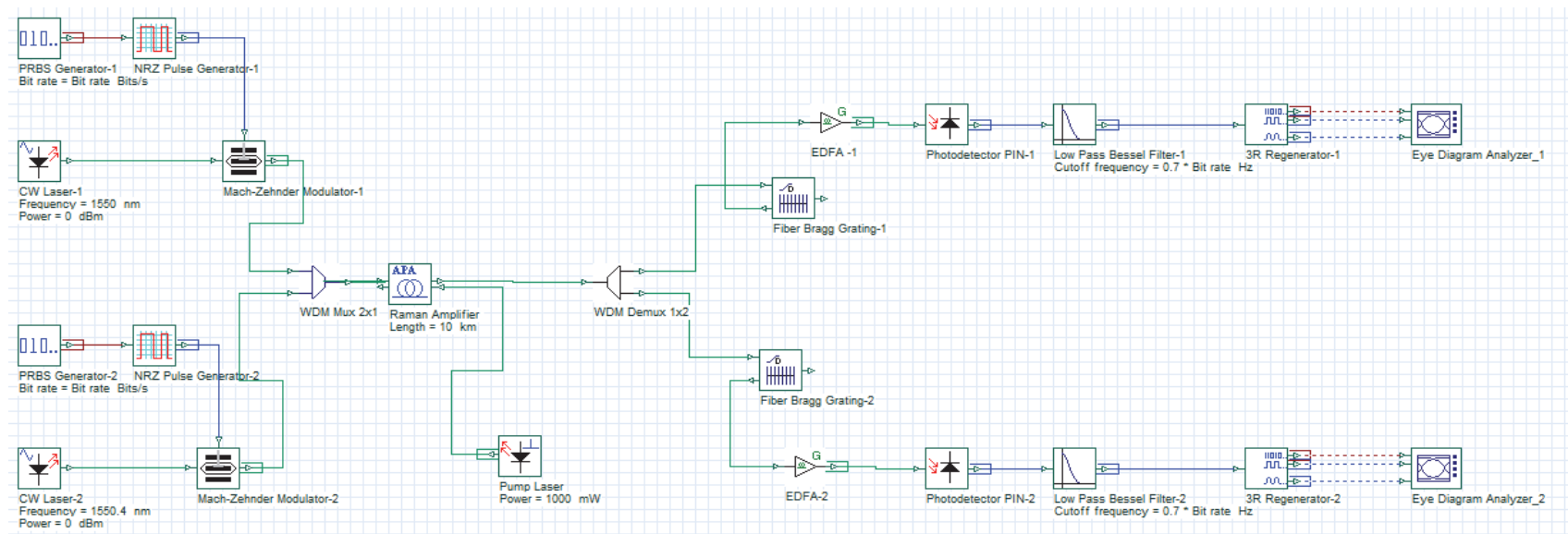

FIGURE 10: Fiber Raman amplifier for WDM system using FBG end EDFA. 


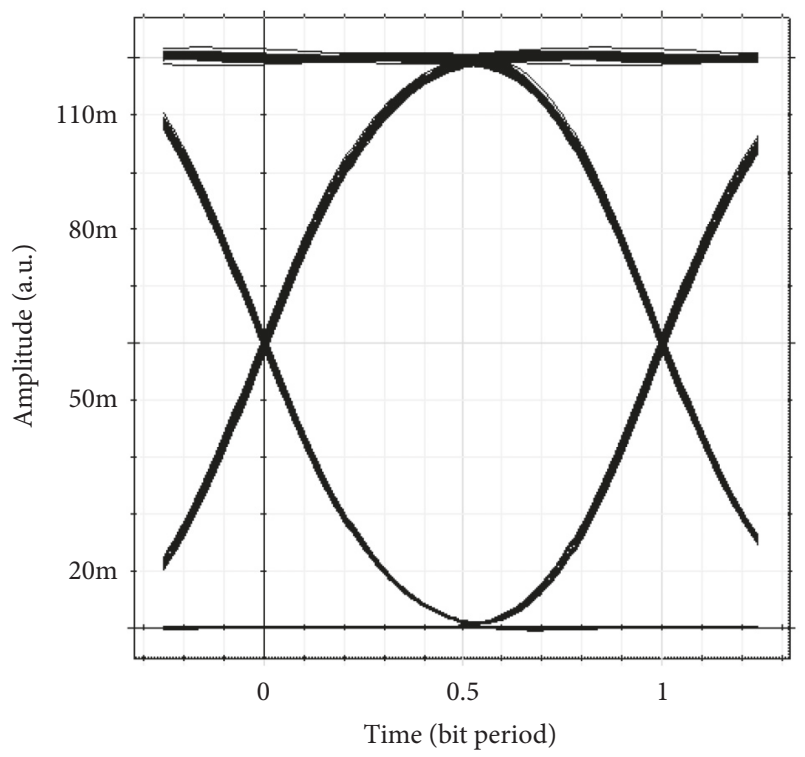

(a)

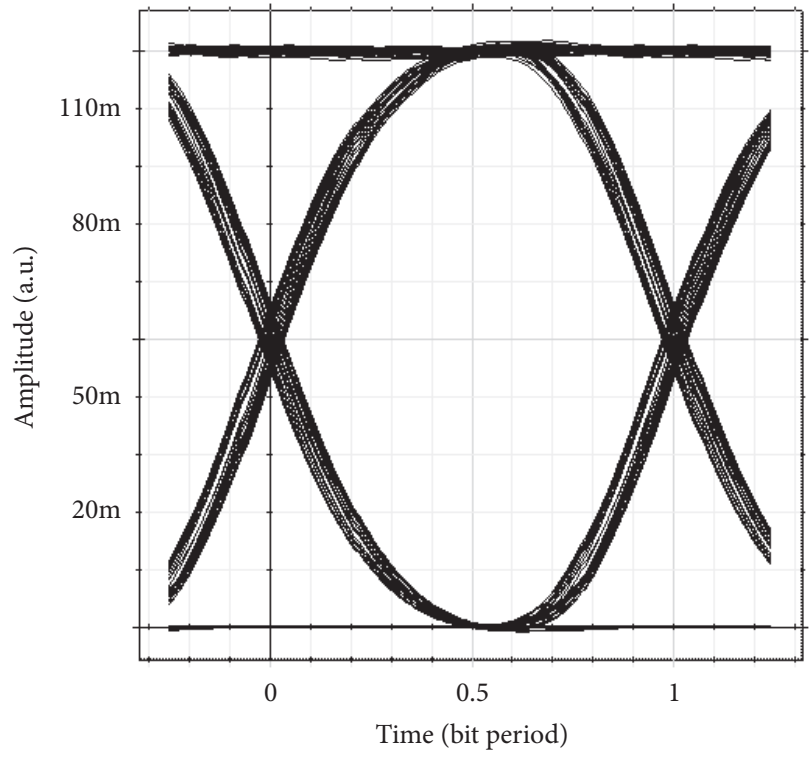

(b)

Figure 11: Eye diagrams for channel spacing in $D=2 \mathrm{ps} / \mathrm{nm} / \mathrm{km}$ : (a) $50 \mathrm{GHz}$; (b) $25 \mathrm{GHz}$.

TABLE 3: Gain, NF, and output power of WDM technology.

\begin{tabular}{lccc}
\hline Channels & Gain $(\mathrm{dB})$ & Noise figure $(\mathrm{dB})$ & Output power $(\mathrm{dBm})$ \\
\hline 1 & 25.7769 & 5.3835 & 16.709 \\
2 & 25.4684 & 5.3660 & 16.752 \\
3 & 25.7332 & 5.3279 & 16.668 \\
4 & 26.5825 & 5.4205 & 16.457 \\
\hline
\end{tabular}

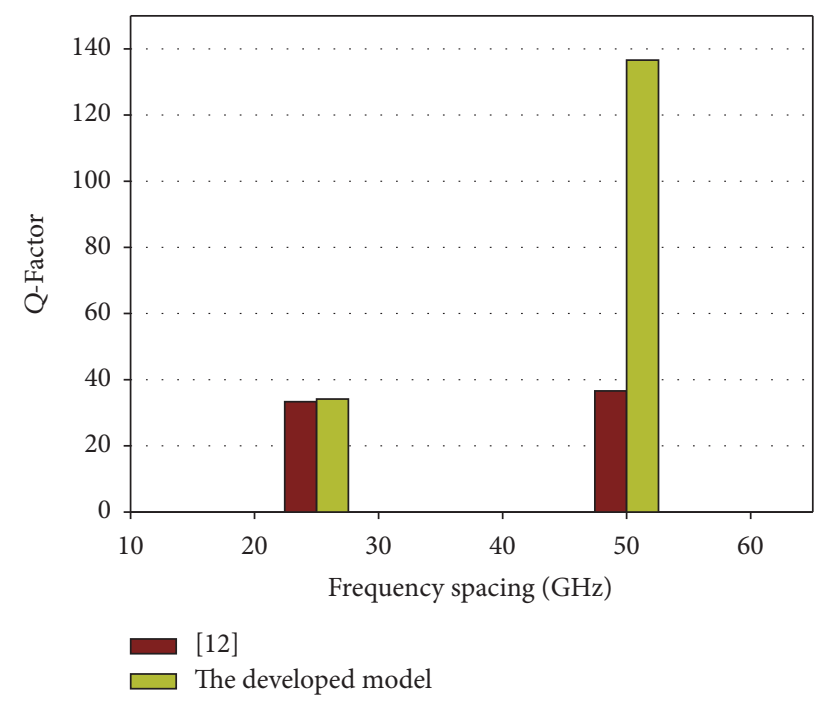

FIGURE 12: Comparison parameters with a previous model and our model.

frequencies [12]. It is clear that, at $25 \mathrm{GHz}$, the value of QFactor is similar for both models, but for the frequency spacing at $50 \mathrm{GHz}$, the Q-Factor in our model is 4 times more important than [12], with efficient received signal quality and lower noise ratio.

\section{Conclusion}

In this paper, a new proposed optical transmission system has been modeled and simulated in order to overcome chromatic dispersion and signal attenuation of optical transmission systems. Firstly, the developed method has been tested in a simple case of one channel transmission to find out the optimum parameter value of the attenuation coefficient. The proposed model has then been adapted in WDM optical transmission system to test the reliability of the developed method. In spite of adapting Wavelength Division Multiplexing technology, results remain valid. The developed model offers reduced signal distortion and improved gain and output power. In other way the model was compared with WDM technology system, results prove that at $50 \mathrm{GHz}$ the Q-Factor of our model is 4 times more important than in [12] in terms of frequency spacing. The new system presents a high efficiency on the received signal quality and lower noise ratio with very satisfactory performances. For future works, the method proposed in this paper can be applied to a complicated system with a big number of channels 
to compensate the chromatic dispersion and decrease the attenuation problems.

\section{Conflicts of Interest}

The authors declare that there are no conflicts of interest regarding the publication of this paper.

\section{References}

[1] N. Antoniades, G. Ellinas, and L. Roudas, "Modeling, Simulation, Design and Engineering of WDM Systems and Networks: An Introduction," in WDM Systems and Networks, Optical Networks, pp. 1-10, Springer, New York, NY, USA, 2012.

[2] R. Ramaswami, K. N. Sivarajan, and G. H. Sasaki, Optical Networks: A Practical Perspective, ELSEVIER, Third Edition, 2010.

[3] M. M. Ismail, M. A. Othman, Z. Zakaria et al., "EDFA-WDM optical network design system," in Proceedings of the Malaysian Technical Universities Conference on Engineering and Technology, MUCET 2012, pp. 294-302, Malaysia, November 2012.

[4] M. Chakkour, A. Hajaji, and O. Aghzout, "Design and Study of EDFA-WDM Optical Transmission System using FBG at 10 Gbits/s Chromatic Despersion Compensation Effects," in Mediterranean Conference on Information \& Communication technologies, May 2015.

[5] M. Othman, "An Analysis of 10 Gbits/s Optical Transmission System using Fiber Bragg Grating (FBG)," IOSR Journal OfEngineering, vol. 02, no. 07, pp. 55-61, 2012.

[6] K. Kumar, "Performance analysis of dispersion compensation using fiber bragg grating (FBG) in optical communication," International Journal of Current Engineering and Technology (IJCET), vol. 4, June 2014.

[7] P. Anuseetal and S. Singh, "Analysis of EDFA based 16-Channel C-Band Optical WDM System for Different Pumping Schemes," International Journal of Advanced Research in Electrical, Electronics and Instrumentation Engineering, vol. 5, no. 6, June 2016.

[8] S. Singh, A. Singh, and R. S. Kaler, "Performance evaluation of EDFA, RAMAN and SOA optical amplifier for WDM systems," ELSEVIER, vol. 124, no. 2, pp. 95-101, 2011.

[9] S. Semmalar and S. Malarkkan, "Output signal power analysis in erbium-doped fiber amplifier with pump ower and length variation using various pumping techniques," ISRN Electronics, vol. 2013, 6 pages, 2013.

[10] R. Kaur and M. Singh, "Dispersion compensation in optical fiber communication system using WDM with DCF and FBG," IOSR Journal of Electronics and Communication Engineering (IOSR-JECE), 2016.

[11] P. Gopika and S. A. Thomas, "Performance analysis of dispersion compensation using FBG and DCF in WDM Systems," International Journal of Advanced Research in Computer and Communication Engineering, vol. 4, no. 10, October 2015.

[12] A. Bindal and S. Singh, "Dispersion analysis of fiber Raman amplifier for WDM system," Elsevier in Optics \& LaserTechnology, vol. 58, pp. 20-25. 

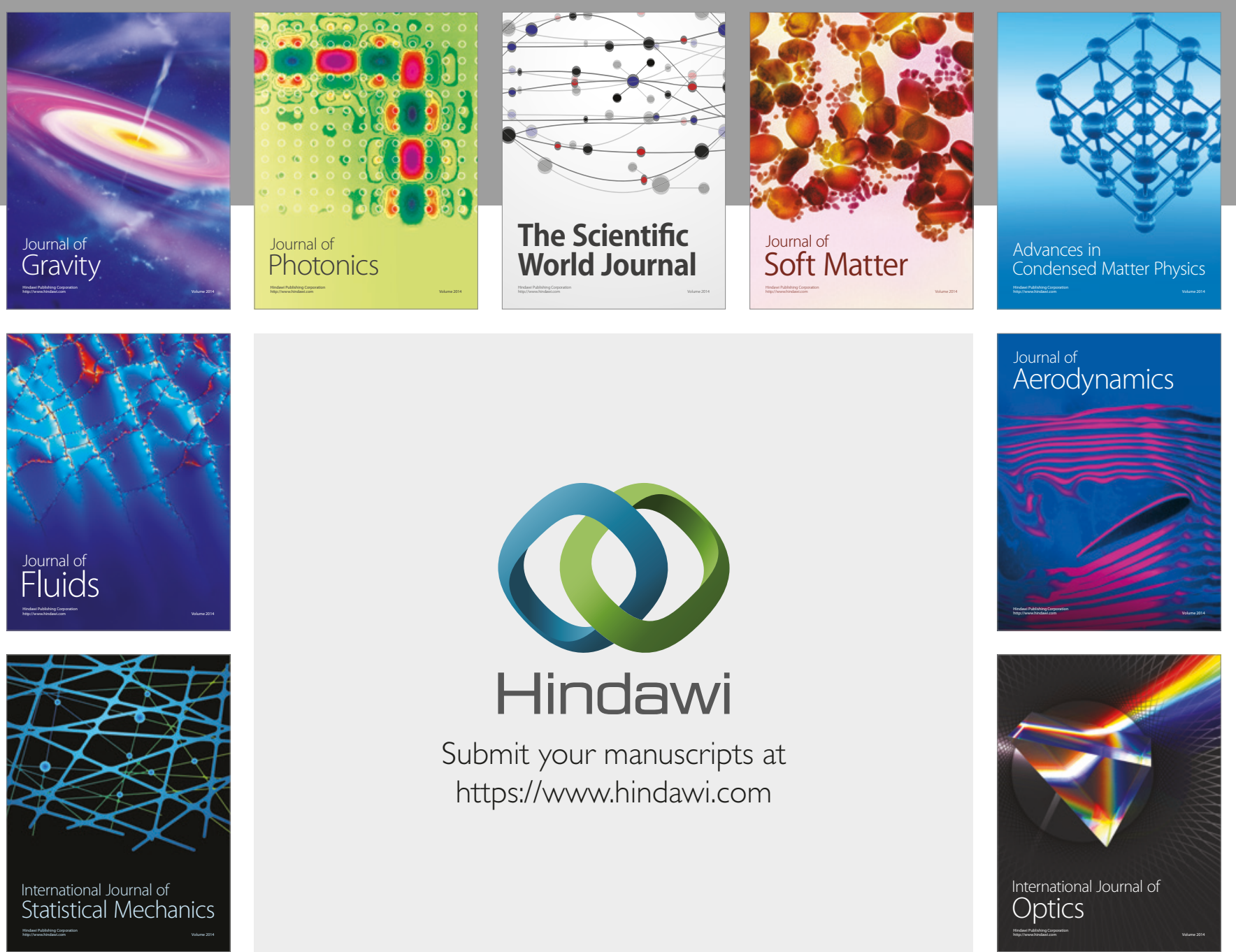

Submit your manuscripts at

https://www.hindawi.com
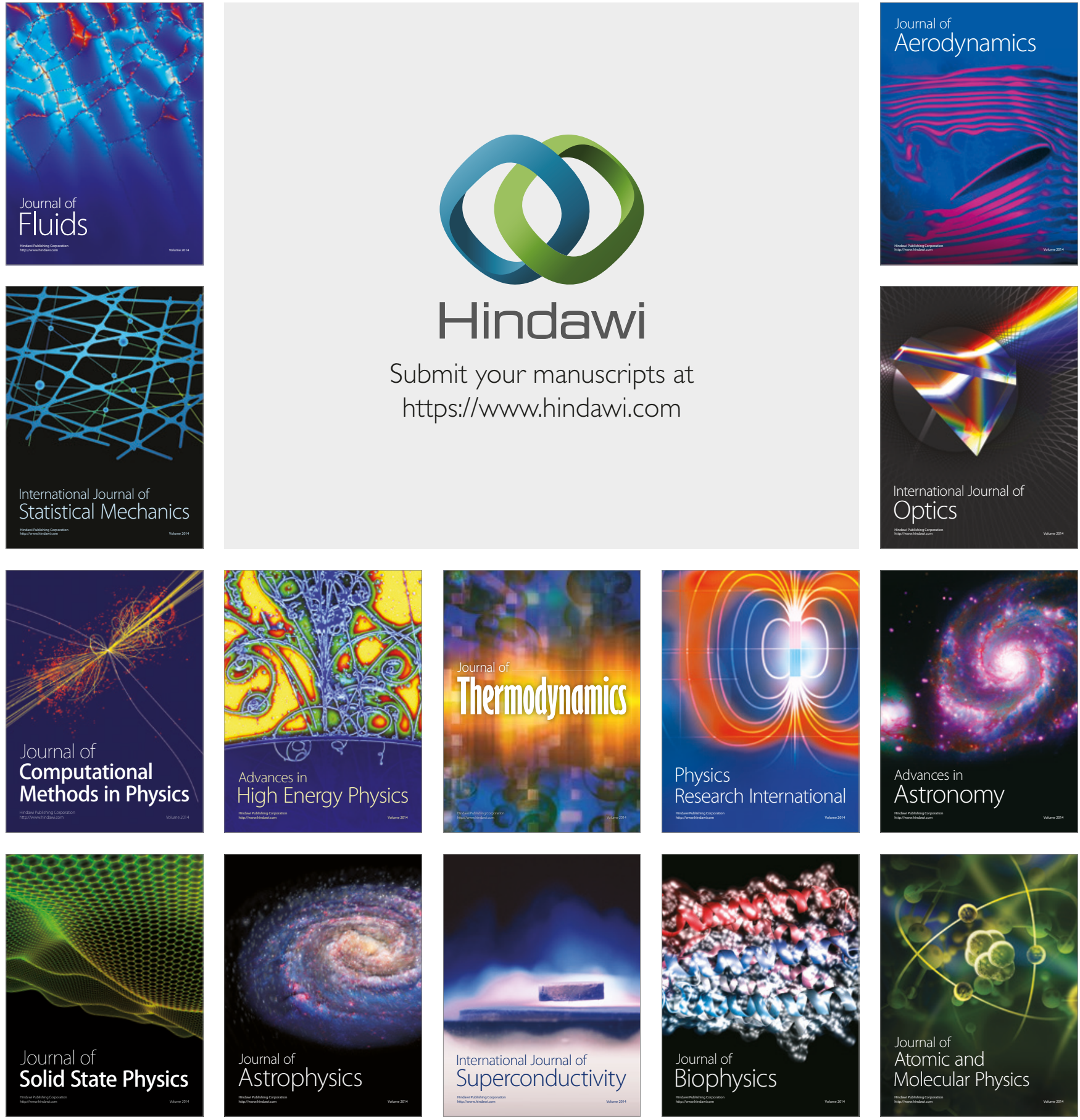